\title{
ON STABILITY OF VELOCITY VECTORS FOR SOME PASSIVE TRACER MODELS
}

\author{
TOMASZ SZAREK, MACIEJ ŚLȨCZKA, AND MARIUSZ URBAŃSKI \\ Dedicated to the memory of Professor Andrzej Lasota (1932-2006)
}

\begin{abstract}
A new result for stability of Markov semigroups is presented. We apply this result to the equation of the passive tracer in a compressible random flow showing that the velocity of a particle converges weakly to some random vector.
\end{abstract}

\section{INTRODUCTION}

The main aim of this paper is to show the utility of lower bound technique in the theory of Markov semigroups acting on measures. For the first time this technique was used by Lasota and Yorke in [10]. The authors proved therein the existence and uniqueness of an absolutely continuous invariant measure for the Frobenius-Perron operator corresponding to piecewise monotonic transformations (see [10]). The methods developed by Lasota and Yorke were applied to Markov operators and semigroups of Markov operators defined on densities (see [9] and the references therein).

Lower bound technique demonstrates its utility in examining Markov semigroups acting on measures as well (see [11]).

Our paper is closely related to paper [8] where we established sufficient conditions for the existence of an invariant measure for Markov semigroups. Here we extend these results and formulate criteria for stability. We also prove some results concerning the sweeping property of a Markov semigroup.

In the second part of our paper these results are applied to general stochastic differential equations. Finally, we show that a good example of application of our results is the equation describing the passive tracer in a compressible random flow. Further references to this equation may be found in [6]. Indeed, applying our results we obtain then that the velocity of the passive tracer converges weakly to some random vector.

\section{MARKov SEMigroups}

Let $(X, \rho)$ be a Polish space and let $\mathcal{B}(X)$ denote the $\sigma$-algebra of Borel sets. By $B_{b}(X)$ we denote the space of bounded Borel-measurable functions equipped with the supremum

2000 Mathematics Subject Classification. Primary: 60J25, 60H15; Secondary: 76N10.

Key words and phrases. Ergodicity of Markov families, invariant measures, stochastic evolution equations, passive tracer dynamics.

This work has been partly supported by Polish Ministry of Science and Higher Education Grants N 201 0211 33. It has been also supported by EU FP6 Marie Curie RTN: CODY. (TS). 
norm. Let $\left(P_{t}\right)_{t \geq 0}$ be the Markovian semigroup defined on $B_{b}(X)$. For each $t \geq 0$ we have $P_{t} \mathbf{1}=\mathbf{1}$ and $P_{t} \psi \geq 0$ if $\psi \geq 0$. Throughout this paper we shall assume that the semigroup is Feller, i.e. $P_{t}\left(C_{b}(X)\right) \subset C_{b}(X)$ for all $t>0$ and stochastically continuous, i.e. for $\psi \in C_{b}(X)$ and $x \in X$ we have $\lim _{t \rightarrow 0+} P_{t} \psi(x)=\psi(x)$. Here $C_{b}(X)$ is the subspace of bounded continuous functions. By $\mathcal{L}_{b}(X)$ we will denote the subspace of bounded Lipschitz functions. Let $\mathcal{M}_{1}$ denote the space of all probability Borel measures on $X$. By supp $\mu$ we denote the support of the measure $\mu$. By $\mathcal{M}_{1}(A)$ for $A \in \mathcal{B}(X)$ we denote the subspace of probability Borel measures that are supported in $A$.

We say that $\mu_{*} \in \mathcal{M}_{1}$ is invariant for $\left(P_{t}\right)_{t \geq 0}$ if

$$
\int_{X} P_{t} \psi(x) \mu_{*}(\mathrm{~d} x)=\int_{X} \psi(x) \mu_{*}(\mathrm{~d} x)
$$

for every $\psi \in B_{b}(X)$ and $t \geq 0$. Alternatively, we can say that $P_{t}^{*} \mu_{*}=\mu_{*}$ for all $t \geq 0$, where $\left(P_{t}^{*}\right)_{t \geq 0}$ denotes the semigroup dual to $\left(P_{t}\right)_{t \geq 0}$, i.e. for a given Borel measure $\mu$ and $t \geq 0$ we set

$$
P_{t}^{*} \mu(A):=\int_{X} P_{t} \mathbf{1}_{A}(x) \mu(\mathrm{d} x) \quad \text { for } A \in \mathcal{B}(X)
$$

Let $\mu_{*} \in \mathcal{M}_{1}$ be an invariant measure for a semigroup $\left(P_{t}\right)_{t \geq 0}$. The semigroup $\left(P_{t}\right)_{t \geq 0}$ is called asymptotically stable if the sequence $\left(P_{t}^{*} \mu\right)_{t \geq 0}$ converges weakly to $\mu_{*}$ for any $\mu \in \mathcal{M}_{1}$. We shall denote this by $\mathrm{w}-\lim _{t \rightarrow \infty} P_{t}^{*} \mu=\mu_{*}$.

We say that a transition semigroup $\left(P_{t}\right)_{t \geq 0}$ has the e-property if the family $\left\{P_{t} \psi\right\}_{t \geq 0}$ is equicontinuous at every point $x$ of $X$ for any bounded and Lipschitz continuous function $\psi$.

We say that a semigroup $\left(P_{t}\right)_{t \geq 0}$ is sweeping from some family $\Xi \subset \mathcal{B}(X)$ if

$$
\lim _{t \rightarrow \infty} P_{t}^{*} \mu(A)=0
$$

for every set $A \in \Xi$ and every measure $\mu \in \mathcal{M}_{1}$.

For a given $t>0$ and $\mu \in \mathcal{M}_{1}$ define $Q^{t} \mu:=t^{-1} \int_{0}^{t} P_{s}^{*} \mu \mathrm{d} s$. When $t=0$ we adopt the convention $Q^{0} \mu:=\mu$. We also write $Q^{t}(x, \cdot)$ in the particular case when $\mu=\delta_{x}$. Let

$$
\mathcal{T}:=\left\{x \in X: \text { the family of measures }\left(Q^{t}(x, \cdot)\right)_{t \geq 0} \text { is tight }\right\} .
$$

Proposition 1. Assume that $\left(P_{t}\right)_{t \geq 0}$ has the e-property. Then the set $\mathcal{T}$ is a $G_{\delta}-$ set. In particular $\mathcal{T}$ is Borel measurable.

Proof. Let $x \in \mathcal{T}$ and let $n \in \mathbb{N}$. Since $\left(Q^{t}(x, \cdot)\right)_{t \geq 0}$ is tight, there is a compact set $K_{x, n} \subset X$ such that

$$
Q^{t}\left(x, K_{x, n}\right)>1-1 / n \quad \text { for } t \geq 0 .
$$

Let $f^{x, n} \in \mathcal{L}_{b}(X)$ be an arbitrary function satisfying

$$
\mathbf{1}_{K_{x, n}} \leq f^{x, n} \leq \mathbf{1}_{K_{x, n}^{1 / n}}
$$


where $K^{\varepsilon}:=\{y \in X:$ dist $(y, K)<\varepsilon\}, \varepsilon>0$, denotes an $\varepsilon$-neighborhood of $K \subset X$. By the e-property we may choose $\delta_{x, n}>0$ such that

$$
\left|P_{t} f^{x, n}(x)-P_{t} f^{x, n}(y)\right|<1 / n \quad \text { for } t \geq 0 \text { and } y \in B\left(x, \delta_{x, n}\right) .
$$

For $y \in B\left(x, \delta_{x, n}\right)$ we have

$$
\begin{aligned}
Q^{t}\left(y, K_{x, n}^{1 / n}\right) & \geq \int_{X} f^{x, n}(z) Q^{t}(y, \mathrm{~d} z)=\frac{1}{t} \int_{0}^{t} P_{s} f^{x, n}(y) \mathrm{d} s \\
& \geq \frac{1}{t} \int_{0}^{t} P_{s} f^{x, n}(x) \mathrm{d} s-1 / n \\
& =\int_{X} f^{x, n}(z) Q^{t}(x, \mathrm{~d} z)-1 / n \\
& \geq Q^{t}\left(x, K_{x, n}\right)-1 / n \geq 1-2 / n \quad \text { for } t \geq 0 .
\end{aligned}
$$

Set

$$
G_{n}=\bigcup_{x \in \mathcal{T}} B\left(x, \delta_{x, n}\right) \quad \text { for } n \geq 1
$$

To finish the proof we show that

$$
\mathcal{T}=\bigcap_{n=1}^{\infty} G_{n} .
$$

Obviously $\mathcal{T} \subset \bigcap_{n=1}^{\infty} G_{n}$. Now let $y \in \bigcap_{n=1}^{\infty} G_{n}$. Then for any $n \in \mathbb{N}$ there exists $y_{n} \in \mathcal{T}$ such that $y \in B\left(y_{n}, \delta_{y_{n}, n}\right)$. From (2.2) it follows that

$$
Q^{t}\left(y, K_{y_{n}}^{1 / n}\right) \geq 1-1 / n \quad \text { for } t \geq 0 .
$$

LeCam's theorem (see [2]) shows that $\left(Q^{t}(y, \cdot)\right)_{t \geq 0}$ is tight. This completes the proof.

Lemma 1. Let $\Gamma^{t}: X \rightarrow 2^{X}, t \geq 0$, be the multifunction given by the formula

$$
\Gamma^{t}(x)=\operatorname{supp} P_{t}^{*} \delta_{x} \quad \text { for } x \in X .
$$

If $\left(P_{t}\right)_{t \geq 0}$ has the e-property, then

$$
\Gamma^{t}(\mathcal{T}) \subset \mathcal{T} \quad \text { for } t \geq 0
$$

Proof. Assume, contrary to our claim, that $\Gamma^{T}(x) \backslash \mathcal{T} \neq \emptyset$ for some $T>0$ and $x \in \mathcal{T}$. Let $y \in \Gamma^{T}(x) \backslash \mathcal{T}$. From the definition of $\mathcal{T}$ and Theorem 3.1 in [12] it follows that there exist a strictly increasing sequence of positive numbers $\left(T_{i}\right)_{i \geq 1}$ satisfying $\lim _{i \rightarrow+\infty} T_{i}=+\infty$, a positive number $\varepsilon$ and a sequence of compact sets $\left(K_{i}\right)_{i \geq 1}$ such that

$$
Q^{T_{i}}\left(x, K_{i}\right) \geq \varepsilon, \quad \forall i \geq 1
$$

and

$$
\min \left\{\rho(x, y): x \in K_{i}, y \in K_{j}\right\} \geq \varepsilon \quad \text { for } i \neq j .
$$

This part of the proof parallels mostly the proof of Proposition 2.1 in [13] but we provide it here in full for the convenience of the reader and since some details are different. Indeed, 
we claim that there exist sequences $\left(\tilde{f}_{n}\right)_{n \geq 1} \subset \mathcal{L}_{b}(X),\left(\nu_{n}\right)_{n \geq 1} \subset \mathcal{M}_{1}(B(x, 1 / n))$ and an increasing sequence of integers $\left(m_{n}\right)_{n \geq 1}$ such that

$$
\mathbf{1}_{K_{m_{n}}} \leq \tilde{f}_{n} \leq \mathbf{1}_{K_{m_{n}}^{\varepsilon / 4}} \text { and } \operatorname{Lip}\left(\tilde{f}_{n}\right) \leq 4 / \varepsilon, \quad \text { for } n \geq 1
$$

Here $\operatorname{Lip}(f):=\sup \left\{|f(x)-f(y)| \rho^{-1}(x, y): x \neq y\right\}$. Moreover,

$$
Q^{t} \nu_{n}\left(\bigcup_{i=n}^{\infty} K_{m_{i}}^{\varepsilon / 4}\right) \leq \varepsilon / 4 \text { for } t \geq 0
$$

and

$$
\left|P_{t} f_{n}(x)-P_{t} f_{n}(y)\right|<\varepsilon / 4 \quad \text { for } t \geq 0, y \in \operatorname{supp} \nu_{n},
$$

$f_{1}:=0$ and $f_{n}:=\sum_{i=1}^{n-1} \tilde{f}_{i}, n \geq 2$. The above is shown by induction on $n$. Let $n=1$. Since $y \in \operatorname{supp} P_{T}^{*} \delta_{x}$, we have $P_{T}^{*} \delta_{x}(B(y, \delta))>0$ for all $\delta>0$. Define the probability measure $\nu_{1}$ by the formula $\nu_{1}(\cdot)=\left(P_{T}^{*} \delta_{x}(B(y, 1))\right)^{-1} P_{T}^{*} \delta_{x}(\cdot \cap B(y, 1))$. Since $\nu_{1} \leq\left(P_{T}^{*} \delta_{x}(B(y, 1))\right)^{-1} P_{T}^{*} \delta_{x}$, from the fact that $x \in \mathcal{T}$ and Lemma 2 in [8] it follows that the family $\left(Q^{t} \nu_{1}\right)_{t \geq 0}$ is tight. Thus, there exists a compact set $K$ such that

$$
Q^{t} \nu_{1}\left(K^{c}\right) \leq \varepsilon / 4 \quad \text { for } \quad t \geq 0 .
$$

Note however that $K \cap K_{i}^{\varepsilon / 4} \neq \emptyset$ only for finitely many $i$-s, by (2.6). Hence there exists an integer $m_{1}$ such that

$$
Q^{t} \nu_{1}\left(\bigcup_{i=m_{1}}^{\infty} K_{i}^{\varepsilon / 4}\right) \leq \varepsilon / 4 \text { for } t \geq 0 .
$$

Let $\tilde{f}_{1}$ be an arbitrary Lipschitz function satisfying $\mathbf{1}_{K_{m_{1}}} \leq \tilde{f}_{1} \leq \mathbf{1}_{K_{m_{1}}^{\varepsilon / 4}}$ and $\operatorname{Lip}\left(\tilde{f}_{1}\right) \leq 4 / \varepsilon$.

Assume now that for a given $n \geq 1$ we have already constructed $\tilde{f}_{1}, \ldots, \tilde{f}_{n}, \nu_{1}, \ldots, \nu_{n}$, $m_{1}, \ldots, m_{n}$ satisfying (2.7)-(2.9). Since $\left(P_{t} f_{n+1}\right)_{t \geq 0}$ is equicontinuous we can choose $\delta<$ $1 /(n+1)$ such that $\left|P_{t} f_{n+1}(x)-P_{t} f_{n+1}(y)\right|<\varepsilon / 4$ for all $t \geq 0$ and $y \in B(x, \delta)$. Suppose furthermore that $\nu_{n+1}:=\left(P_{T}^{*} \delta_{x}(B(x, \delta))\right)^{-1} P_{T}^{*} \delta_{x}(\cdot \cap B(x, \delta))$. Since the measure is supported in $B(x, \delta)$ condition (2.9) holds with $f_{n+1}$ in place of $f_{n}$ and $\nu_{n+1}$ in place of $\nu_{n}$. Tightness of $\left(Q^{t} \nu_{n+1}\right)_{t \geq 0}$ can be argued in the same way as in case $n=1$. In consequence, one can find $m_{n+1}>m_{n}$ such that

$$
Q^{t} \nu_{n+1}\left(\bigcup_{i=m_{n+1}}^{\infty} K_{i}^{\varepsilon / 4}\right) \leq \varepsilon / 4 \quad \text { for } t \geq 0 .
$$

Finally, we let $\tilde{f}_{n+1}$ be an arbitrary continuous function satisfying (2.7).

Observe that conditions (2.6) and (2.7) imply that the series $f:=\sum_{i=1}^{\infty} \tilde{f}_{i}$ is uniformly convergent and $f \in \mathcal{L}_{b}(X)$. We easily check (see the argument given in [8]) that

$$
\int_{X} Q^{T_{m_{n}}}(y, \mathrm{~d} w) f(w)-\int_{X} Q^{T_{m_{n}}} \nu_{n}(\mathrm{~d} w) f(w)>\varepsilon / 2 .
$$


Hence, there must be a sequence $\left(t_{n}, y_{n}\right)$ such that $t_{n} \in\left[0, T_{m_{n}}\right], y_{n} \in \operatorname{supp} \nu_{n} \subset B(x, 1 / n)$ for which $P_{t_{n}} f(y)-P_{t_{n}} f\left(y_{n}\right)>\varepsilon / 2, n \geq 1$. This clearly contradicts equicontinuity of $\left(P_{t} f\right)_{t \geq 0}$ at the point $y$.

Remark 1. From the proof of Lemma 1 it follows that if $\left(Q^{t} \mu\right)_{t \geq 0}$ is tight for some $\mu \in \mathcal{M}_{1}$, then $\operatorname{supp} \mu \subset \mathcal{T}$.

In [8] it has been proved the following theorem.

Theorem 1. Assume that $\left(P_{t}\right)_{t \geq 0}$ has the e-property and that there exists $z \in X$ such that for every $\delta>0$ and $x \in X$,

$$
\liminf _{t \rightarrow \infty} Q^{t}(x, B(z, \delta))>0 .
$$

Then $\left(P_{t}\right)_{t \geq 0}$ admits a unique invariant probability measure $\mu_{*}$. Moreover

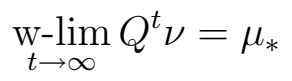

for any $\nu \in \mathcal{M}_{1}$ that is supported in $\mathcal{T}$.

In [8] we have also provided an example showing that the set $\mathcal{T}$ for the semigroup $\left(P_{t}\right)_{t \geq 0}$ satisfying the assumptions of the above theorem may not be the entire space $X$.

Remark 2. From the above theorem it follows that if a probability measure $\nu$ is supported in $\mathcal{T}$, then $\left(Q^{t} \nu\right)_{t \geq 0}$ is weakly convergent. Hence $\left(Q^{t} \nu\right)_{t \geq 0}$ is tight, by Alexandrov's theorem.

Remark 3. Let $\left(P_{t}\right)_{t \geq 0}$ satisfy the assumptions of Theorem 1 and let $\mu_{*}$ be its invariant measure. Then $z \in \operatorname{supp} \mu_{*}$. Indeed, by Fatou's lemma and (2.11) we have

$$
\begin{aligned}
\mu_{*}(B(z, r)) & =\liminf _{t \rightarrow \infty} Q^{t} \mu_{*}(B(z, r))=\liminf _{t \rightarrow \infty} \int_{X} Q^{t} \delta_{x}(B(z, r)) \mu_{*}(\mathrm{~d} x) \\
& \geq \int_{X} \liminf _{t \rightarrow \infty} Q^{t} \delta_{x}(B(z, r)) \mu_{*}(\mathrm{~d} x)>0 \quad \text { for any } r>0 .
\end{aligned}
$$

Proposition 2. If $\left(P_{t}\right)_{t \geq 0}$ satisfies the assumption of Theorem 1, then the set $\mathcal{T}$ given by (2.1) is closed.

Proof. Assume, contrary to our claim, that $x \in \operatorname{cl} \mathcal{T} \backslash \mathcal{T}$. Since $\left(Q^{t}(x, \cdot)\right)_{t \geq 0}$ is not tight, there exists (see Theorem 3.1 in [12]) a strictly increasing sequence of positive numbers $\left(T_{i}\right)_{i \geq 1}$ with $\lim _{i \rightarrow+\infty} T_{i}=+\infty$, a positive numbers $\varepsilon$ and a sequence of compact sets $\left(K_{i}\right)_{i \geq 1}$ such that

$$
Q^{T_{i}}\left(x, K_{i}\right) \geq \varepsilon \quad \text { for all } i \geq 1
$$

and

$$
\min \left\{\rho(x, y): x \in K_{i}, y \in K_{j}\right\} \geq \varepsilon \quad \text { for } i \neq j .
$$

Let $\mu_{*}$ be a unique probability measure for $\left(P_{t}\right)_{t \geq 0}$, by Theorem 1. By Ulam's lemma we may choose a compact set $K \subset X$ such that

$$
\mu_{*}(K)>1-\varepsilon / 2 \text {. }
$$


Since $K$ is compact, there exists $N_{0} \in \mathbb{N}$ such that

$$
K_{i}^{\varepsilon / 3} \cap K=\emptyset \quad \text { for } i \geq N_{0} .
$$

Further, let $f$ be an arbitrary Lipschitz function such that

$$
\sum_{i=N_{0}}^{\infty} \mathbf{1}_{K_{i}}(x) \leq f(x) \leq \sum_{i=N_{0}}^{\infty} \mathbf{1}_{K_{i}^{\varepsilon / 3}}(x) \text { for } x \in X .
$$

Since $\left(P_{t} f\right)_{t \geq 0}$ is equicontinuous, we may choose $r>0$ such that

$$
\left|P_{t} f(x)-P_{t} f(y)\right|<\varepsilon / 2 \quad \text { for } y \in B(x, r) \text { and } t \geq 0 .
$$

Since $x \in \operatorname{cl} \mathcal{T}$, we have $\mathcal{T} \cap B(x, r) \neq \emptyset$. Fix $y \in \mathcal{T} \cap B(x, r)$. From (2.13) it follows that

$$
Q^{T_{i}}\left(x, \bigcup_{i=N_{0}}^{\infty} K_{i}\right) \geq \varepsilon \quad \text { for } i \geq N_{0} \text {. }
$$

By the definition of $f$ we obtain then

$$
\frac{1}{T_{i}} \int_{0}^{T_{i}} P_{s} f(x) \mathrm{d} s=\int_{X} f(y) Q^{T_{i}}(x, \mathrm{~d} y) \geq Q^{T_{i}}\left(x, \bigcup_{i=N_{0}}^{\infty} K_{i}\right) \geq \varepsilon .
$$

Consequently, by (2.18) we have

$$
\begin{aligned}
\frac{1}{T_{i}} \int_{0}^{T_{i}} P_{s} f(y) \mathrm{d} s & \geq \frac{1}{T_{i}} \int_{0}^{T_{i}} P_{s} f(x) \mathrm{d} s-\frac{1}{T_{i}} \int_{0}^{T_{i}}\left|P_{s} f(x)-P_{s} f(y)\right| \mathrm{d} s \\
& \geq \varepsilon-\varepsilon / 2=\varepsilon / 2
\end{aligned}
$$

which, in turn, by (2.16) gives

$$
Q^{T_{i}}\left(y, \bigcup_{i=N_{0}+1}^{\infty} K_{i}^{\varepsilon / 6}\right) \geq \frac{1}{T_{i}} \int_{0}^{T_{i}} P_{s} f(y) \mathrm{d} s>\varepsilon / 2 .
$$

On the other hand, from the fact that $\mathrm{w}-\lim _{t \rightarrow \infty} Q^{t} \delta_{y}=\mu_{*}$, it follows that

$$
\liminf _{t \rightarrow \infty} Q^{t}\left(y, K^{\varepsilon / 6}\right) \geq \mu_{*}(K)>1-\varepsilon / 2,
$$

by the Alexandrov theorem and condition (2.15). Thus, by (2.16) we have

$$
\begin{aligned}
& \limsup _{t \rightarrow \infty} Q^{t}\left(y, \bigcup_{i=N_{0}}^{\infty} K_{i}^{\varepsilon / 6}\right) \leq \limsup _{t \rightarrow \infty} Q^{t}\left(y, X \backslash K^{\varepsilon / 6}\right) \\
& \leq 1-\liminf _{t \rightarrow \infty} Q^{t}\left(y, K^{\varepsilon / 6}\right) \leq 1-(1-\varepsilon / 2)=\varepsilon / 2
\end{aligned}
$$

which contradicts condition (2.19). This finishes the proof.

The following lemma is a straightforward application of the Arzela-Ascoli theorem. 
Lemma 2. Let $\mathcal{F}$ be a family of uniformly bounded and equicontinuous functions. Then

$$
\sup _{f \in \mathcal{F}}\left|\int_{X} f \mathrm{~d} \mu_{n}-\int_{X} f \mathrm{~d} \mu_{*}\right| \rightarrow 0 \quad \text { as } n \rightarrow \infty,
$$

provided $\mathrm{w}-\lim _{n \rightarrow \infty} \mu_{n}=\mu_{*}$ for $\mu_{n}, \mu_{*} \in \mathcal{M}_{1}$.

The proof is standard and we leave it for the reader. Indeed, it is enough to observe that by the Prokhorov theorem (see [1]) we may assume, without loss of generality, that $X$ is compact. Application of the Arzela-Ascoli theorem finishes then the proof.

For a given closed set $A \subset X$ and positive constants $\eta$ and $\alpha$ with $\alpha<1$, we set

$$
\mathcal{M}_{\alpha}^{\eta}(A):=\left\{\mu \in \mathcal{M}_{1}: \exists \gamma<\eta \liminf _{t \rightarrow \infty} P_{t}^{*} \mu\left(A^{\gamma}\right)>1-\alpha\right\}
$$

Observe that $\mathcal{M}_{\alpha}^{\eta}(A)$ is a convex set and

$$
P_{t}^{*}\left(\mathcal{M}_{\alpha}^{\eta}(A)\right) \subset \mathcal{M}_{\alpha}^{\eta}(A) \quad \text { for all } t \geq 0
$$

The following lemma will be useful in the sequel.

Lemma 3. Let $\left(P_{t}\right)_{t \geq 0}$ have the e-property. Then for every closed set $A \subset X$ and positive constants $\eta, \alpha$ with $\alpha<1$, the set $\mathcal{M}_{\alpha}^{\eta}(A)$ is open in the weak topology of $\mathcal{M}_{1}$.

Proof. Let positive constants $\eta, \alpha$ with $\alpha<1$ and a closed set $A \subset X$ be given. Fix $\mu \in \mathcal{M}_{\alpha}^{\eta}(A)$. To finish the proof it is enough to show that for every sequence of probability Borel measures $\left(\mu_{n}\right)_{n \geq 1}$ that converges weakly to $\mu$, we have $\mu_{n} \in \mathcal{M}_{\alpha}^{\eta}(A)$ for all $n$ large enough. By the definition of the set $\mathcal{M}_{\alpha}^{\eta}(A)$ there exists $\gamma<\eta$ such that

$$
\liminf _{t \rightarrow \infty} P_{t}^{*} \mu\left(A^{\gamma}\right)>1-\alpha
$$

Choose $\sigma>0$ such that $\gamma+\sigma<\eta$ and let $\varphi$ be a Lipschitz function such that

$$
\mathbf{1}_{A^{\gamma}}(x) \leq \varphi(x) \leq \mathbf{1}_{A^{\gamma+\sigma}}(x) \quad \text { for all } x \in X .
$$

Fix $\left(\mu_{n}\right)_{n \geq 1}$ such that $\mathrm{w}-\lim _{n \rightarrow \infty} \mu_{n}=\mu$. Since $\left(P_{t}\right)_{t \geq 0}$ has the e-property, the family $\left\{P_{t} \varphi\right\}_{t \geq 0}$ is equicontinuous and

$$
\sup _{t \geq 0}\left|\int_{X} P^{t} \varphi \mathrm{d} \mu_{n}-\int_{X} P^{t} \varphi \mathrm{d} \mu\right| \rightarrow 0
$$

by Lemma 2. On the other hand, we know that

$$
\begin{aligned}
\int_{X} P_{t} \varphi \mathrm{d} \mu & =\int_{X} \varphi \mathrm{d} P_{t}^{*} \mu \geq \int_{X} \mathbf{1}_{A^{\gamma}} \mathrm{d} P_{t}^{*} \mu \\
& \geq P_{t}^{*} \mu\left(A^{\gamma}\right) \geq 1-\alpha+\Theta
\end{aligned}
$$

for $t$ large enough and some $\Theta>0$. From (2.21) and (2.22) it follows that

$$
\int_{X} P_{t} \varphi \mathrm{d} \mu_{n}>1-\alpha+\Theta / 2
$$


for $n$ and $t$ large enough. Since

$$
\int_{X} P_{t} \varphi \mathrm{d} \mu_{n}=\int_{X} \varphi \mathrm{d} P_{t}^{*} \mu_{n} \leq P_{t}^{*} \mu_{n}\left(A^{\eta+\gamma}\right),
$$

we finally obtain

$$
\liminf _{t \rightarrow \infty} P_{t}^{*} \mu_{n}\left(A^{\eta+\gamma}\right)>1-\alpha
$$

for $n$ large enough. This completes the proof.

Proposition 3. If $\left(P_{t}\right)_{t \geq 0}$ satisfies the assumptions of Theorem 1, then $\left(P_{t}\right)_{t \geq 0}$ is sweeping from compact sets disjoint from $\mathcal{T}$.

Proof. Assume, contrary to our claim, that there exists a compact set $K$ disjoint from $\mathcal{T}$, a positive constant $\alpha$ and a probability measure $\mu$ such that

$$
\limsup _{t \rightarrow \infty} P_{t}^{*} \mu(K)>\alpha .
$$

Since the assumptions of Proposition 2 are satisfied, $\mathcal{T}$ is closed. Therefore there exists $\eta>0$ such that

$$
\inf \{\rho(x, y): x \in K, y \in \mathcal{T}\}>\eta .
$$

Set $\widetilde{\mathcal{M}}:=\mathcal{M}_{\alpha / 2}^{\eta}(\mathcal{T})$ and observe that $\widetilde{\mathcal{M}} \neq \emptyset$. Indeed, from Lemma 1 we obtain that $\delta_{z} \in \widetilde{\mathcal{M}}$. Since $\widetilde{\mathcal{M}}$ is open in the weak topology, by Lemma 3 there exists $\sigma>0$ such that $\nu \in \widetilde{\mathcal{M}}$ provided that $\operatorname{supp} \nu \subset B(z, \sigma)$.

Let $x \in K$. Since $\lim _{\inf _{t \rightarrow \infty}} Q^{t}(x, B(z, \sigma / 2))>0$, there exists $t_{x}>0$ such that $\alpha_{x}:=$ $P_{t_{x}}^{*} \delta_{x}(B(z, \sigma / 2))>0$. Further, since $P_{t_{*}^{*}}^{*}$ is a Feller operator, there exists $r_{x}>0$ such that $P_{t_{x}}^{*} \delta_{y}(B(z, \sigma / 2))>\alpha_{x} / 2$. Obviously,

$$
K \subset \bigcup_{x \in K} B\left(x, r_{x}\right)
$$

and since $K$ is compact, there exist, say, $x_{1}, \ldots, x_{m} \in K$ such that

$$
K \subset \bigcup_{i=1}^{m} B\left(x_{i}, r_{x_{i}}\right) \text {. }
$$

Set $\Theta:=\min _{1 \leq i \leq m} \alpha_{x_{i}} / 2$. Define the constant

$$
\gamma:=\sup \left\{\beta \geq 0: P_{t_{0}}^{*} \mu \geq \beta \nu \text { for some } \nu \in \widetilde{\mathcal{M}} \text { and } t_{0}>0\right\} .
$$

Choose $\nu \in \widetilde{\mathcal{M}}$ and $t_{0}>0$ such that $P_{t_{0}}^{*} \mu \geq \beta \nu$ holds with $\beta>\gamma-\Theta \alpha /(2 m)$. Observe that if $P_{t_{0}}^{*} \mu \geq \beta \nu$ for some $\nu \in \widetilde{\mathcal{M}}$, then from (2.20) it follows that $P_{t}^{*} \mu \geq \beta \nu_{t}$ for $t \geq t_{0}$ with some $\nu_{t} \in \widetilde{\mathcal{M}}$. Therefore we may assume that $P_{t_{0}}^{*} \mu(K)>\alpha$, by $(2.23)$, and consequently

$$
\left(P_{t_{0}}^{*} \mu-\beta \nu\right)(K) \geq \alpha-\alpha / 2=\alpha / 2 \text {. }
$$

Hence there exists $j \in\{1, \ldots, m\}$ such that

$$
\left(P_{t_{0}}^{*} \mu-\beta \nu\right)\left(B\left(x_{j}, r_{x_{j}}\right)\right) \geq \alpha /(2 m) \text {. }
$$


Further, we have

$$
\begin{aligned}
& P_{t_{x_{j}}}^{*}\left(P_{t_{0}}^{*} \mu-\beta \nu\right)(B(z, \sigma))=\int_{X} \mathbf{1}_{B(z, \sigma)}(x)\left(P_{t_{x_{j}}+t_{0}}^{*} \mu-\beta P_{t_{x_{j}}}^{*} \nu\right)(\mathrm{d} x) \\
& =\int_{X} P_{t_{x_{j}}} \mathbf{1}_{B(z, \sigma)}(x)\left(P_{t_{0}}^{*} \mu-\beta \nu\right)(\mathrm{d} x)=\int P_{t_{x_{j}}}^{*} \delta_{x}(B(z, \sigma))\left(P_{t_{0}}^{*} \mu-\beta \nu\right)(\mathrm{d} x) \\
& \geq \int_{B\left(x_{j}, r_{x_{j}}\right)} P_{t_{x_{j}}}^{*} \delta_{x}(B(z, \sigma))\left(P_{t_{0}}^{*} \mu-\beta \nu\right)(\mathrm{d} x) \geq \Theta \alpha /(2 m) .
\end{aligned}
$$

Set

$$
\tilde{\nu}(\cdot)=\frac{\left(P_{t_{x_{j}}+t_{0}}^{*} \mu-\beta P_{t_{x_{j}}}^{*} \nu\right)(\cdot \cap B(z, \sigma))}{\left(P_{t_{x_{j}}+t_{0}}^{*} \mu-\beta P_{t_{x_{j}}}^{*} \nu\right)(B(z, \sigma))}
$$

and observe that $\tilde{\nu} \in \widetilde{\mathcal{M}}$, since $\operatorname{supp} \tilde{\nu} \subset B(z, \sigma)$. Let

$$
\hat{\nu}=\beta(\beta+\Theta \alpha /(2 m))^{-1} P_{t_{x_{j}}}^{*} \nu+\Theta \alpha /(2 m)(\beta+\Theta \alpha /(2 m))^{-1} \tilde{\nu} .
$$

Since $P_{t_{x_{j}}}^{*} \nu, \tilde{\nu} \in \widetilde{\mathcal{M}}$ and $\widetilde{\mathcal{M}}$ is convex, we obtain that $\hat{\nu} \in \widetilde{\mathcal{M}}$. Further

$$
P_{t_{x_{j}}+t_{0}}^{*} \mu \geq(\beta+\Theta \alpha /(2 m)) \hat{\nu},
$$

which is impossible as $\beta+\Theta \alpha /(2 m)>\gamma$. This completes the proof.

Our note is aimed at proving the following generalization of Theorem 1.

Theorem 2. Let $\left(P_{t}\right)_{t \geq 0}$ satisfy the assumptions of Theorem 1 and let $\mu_{*}$ be its invariant measure. Assume also that

$$
\liminf _{t \rightarrow \infty} P_{t}^{*} \delta_{z}(B(z, \delta))>0
$$

with $z$ such as in Theorem 1. Then

$$
\mathrm{w}_{t \rightarrow \infty} \lim _{t}^{*} \nu=\mu_{*}
$$

for any $\nu \in \mathcal{M}_{1}$ that is supported in $\mathcal{T}$.

Proof. We divide the proof into two steps.

Step I: We are going to show that for every $\delta>0$ there exists $\alpha>0$ such that

$$
\liminf _{t \rightarrow \infty} P_{t}^{*} \nu(B(z, \delta))>\alpha
$$

for every $\nu \in \mathcal{M}_{1}$ supported in $\mathcal{T}$. To do this fix $\delta>0$. From the e-property it follows that there exists $\eta>0$ such tht

$$
\liminf _{t \rightarrow \infty} P_{t}^{*} \delta_{y}(B(z, \delta))>\frac{1}{2} \liminf _{t \rightarrow \infty} P_{t}^{*} \delta_{z}(B(z, \delta / 2)) \quad \text { for } y \in B(z, \eta) .
$$

It is shown in the same way as condition (3.42) in Lemma 4 in [8]. Set

$$
\gamma:=\frac{1}{2} \liminf _{t \rightarrow \infty} P_{t}^{*} \delta_{z}(B(z, \delta / 2)) \quad \text { and } \quad \vartheta:=\mu_{*}(B(z, \eta)) / 2
$$


From condition (2.24) and Remark 2, we obtain $\gamma, \vartheta>0$. To finish this part of the proof we show that condition (2.26) holds with $\alpha=\gamma \vartheta$. Fix $\nu \in \mathcal{M}_{1}$ supported in $\mathcal{T}$. From Theorem 1 and Alexandrov's theorem we have

$$
\liminf _{t \rightarrow \infty} Q^{t} \nu(B(z, \eta)) \geq \mu_{*}(B(z, \eta)) .
$$

Hence there exists $t_{0}>0$ such that

$$
P_{t_{0}}^{*} \nu(B(z, \eta))>\vartheta
$$

Consequently,

$$
\begin{aligned}
& \liminf _{t \rightarrow \infty} P_{t}^{*} \nu(B(z, \delta))=\liminf _{t \rightarrow \infty} P_{t+t_{0}}^{*} \nu(B(z, \delta)) \\
& \stackrel{\text { Fatou's lemma }}{\geq} \int_{X} \liminf _{t \rightarrow \infty} P_{t}^{*} \delta_{x}(B(z, \delta)) P_{t_{0}}^{*} \nu(\mathrm{d} x) \\
& \geq \int_{B(z, \eta))} \liminf _{t \rightarrow \infty} P_{t}^{*} \delta_{x}(B(z, \delta)) P_{t_{0}}^{*} \nu(\mathrm{d} x)>\gamma \vartheta=\alpha .
\end{aligned}
$$

Since $\nu$ was an arbitrary probability measure supported in $\mathcal{T}$, Step I is complete.

Step II: To finish the proof we will show that

$$
\limsup _{t \rightarrow \infty}\left|\int_{X} P_{t} f(x) \nu_{1}(\mathrm{~d} x)-\int_{X} P_{t} f(x) \nu_{2}(\mathrm{~d} x)\right|=0
$$

for any $f \in \mathcal{L}_{b}(X)$ and $\nu_{1}, \nu_{2} \in \mathcal{M}_{1}$ supported in $\mathcal{T}$. Fix an $\varepsilon>0$ and let $f \in \mathcal{L}_{b}(X)$. By the e-property we may find $\gamma>0$ such that

$$
\left|P_{t} f\left(y_{1}\right)-P_{t} f\left(y_{2}\right)\right|<\varepsilon / 2
$$

for $t \geq 0$ and $y_{1}, y_{2} \in B(z, \gamma)$. Fix $\nu_{1}, \nu_{2} \in \mathcal{M}_{1}$ that are supported in $\mathcal{T}$. From Step I we may choose $\alpha>0$ such that condition (2.26) holds. By induction we will define a sequence of positve reals $\left(t_{i}\right)_{i \geq 0}$ and four sequences of probability measures $\left(\nu_{1}^{i}\right)_{i \geq 0},\left(\nu_{2}^{i}\right)_{i \geq 0},\left(\mu_{1}^{i}\right)_{i \geq 0}$, $\left(\mu_{2}^{i}\right)_{i \geq 0}$ such that $\operatorname{supp} \nu_{i}^{k} \subset B(z, \gamma)$, supp $\mu_{i}^{k} \subset \mathcal{T}$ for $k=1,2, i \geq 1$ and

$$
P_{t_{k}}^{*} \mu_{i}^{k-1}=\alpha \nu_{i}^{k}+(1-\alpha) \mu_{i}^{k} \quad \text { for } i=1,2 \text { and } k \geq 1 .
$$

If $k=0$, we set $t_{0}=0, \nu_{1}^{1}=\mu_{1}^{1}=\nu_{1}$ and $\nu_{2}^{1}=\mu_{2}^{1}=\nu_{2}$. If $k \geq 1$ and $t_{k-1}, \nu_{1}^{k-1}, \nu_{2}^{k-1}$, $\mu_{1}^{k-1}, \mu_{2}^{k-1}$ are given, by Step I and the fact that $\operatorname{supp} \mu_{i}^{k-1} \subset \mathcal{T}, i=1,2$, we may choose $t_{k}>0$ such that

$$
P_{t_{k}}^{*} \mu_{i}^{k-1}(B(z, \gamma))>\alpha \quad \text { for } i=1,2
$$

Now we define

$$
\begin{gathered}
\nu_{i}^{k}(\cdot)=\frac{P_{t_{k}}^{*} \mu_{i}^{k-1}(\cdot \cap B(z, \gamma))}{P_{t_{k}}^{*} \mu_{i}^{k-1}(B(z, \gamma))} \quad \text { for } i=1,2, \\
\mu_{i}^{k}(\cdot)=\frac{1}{1-\alpha}\left(P_{t_{k}}^{*} \mu_{i}^{k-1}(\cdot)-\alpha \nu_{i}^{k}(\cdot)\right) \quad \text { for } i=1,2 .
\end{gathered}
$$


From (2.32) it follows that $\mu_{i}^{k} \in \mathcal{M}_{1}$. Further $\operatorname{supp} \nu_{i}^{k} \subset B(z, \gamma)$ for $i=1,2$. Obviously, condition (2.31) holds. Hence supp $\mu_{i}^{k} \subset \operatorname{supp} P_{t_{k}}^{*} \mu_{i}^{k-1}$. On the other hand, from Lemma 1 and Proposition 2 it follows that $\operatorname{supp} P_{t_{k}}^{*} \mu_{i}^{k-1} \subset \mathcal{T}$ for $i=1,2$ and consequently $\operatorname{supp} \mu_{i}^{k} \subset$ $\mathcal{T}$ for $i=1,2$.

Using (2.32) it is easy to verify that

$$
\begin{aligned}
P_{t_{1}+\ldots+t_{k}+t}^{*} \nu_{i} & =\alpha P_{t_{2}+\ldots+t_{k}+t}^{*} \nu_{i}^{1}+\alpha(1-\alpha) P_{t_{3}+\ldots+t_{k}+t}^{*} \nu_{i}^{2} \\
& +\ldots+\alpha(1-\alpha)^{k-1} P_{t}^{*} \nu_{i}^{k}+(1-\alpha)^{k} P_{t}^{*} \mu_{i}^{k}
\end{aligned}
$$

for $i=1,2, k \geq 1$ and $t \geq 0$. Since

$$
\left|\int_{X} f(y) P_{t}^{*} \nu_{1}^{k}(\mathrm{~d} y)-\int_{X} f(y) P_{t}^{*} \nu_{2}^{k}(\mathrm{~d} y)\right|=\left|\int_{X} P_{t} f(y) \nu_{1}^{k}(\mathrm{~d} y)-\int_{X} P_{t} f(y) \nu_{2}^{k}(\mathrm{~d} y)\right| \leq \varepsilon / 2,
$$

for all $t \geq 0$, by (2.30), we conclude from (2.35) that

$$
\limsup _{t \rightarrow \infty}\left|\int_{X} P_{t} f(x) \nu_{1}(\mathrm{~d} x)-\int_{X} P_{t} f(x) \nu_{2}(\mathrm{~d} x)\right| \leq \varepsilon
$$

Since $\varepsilon>0$ was arbitrary, this finishes Step II. The proof is complete.

\section{AN APPLICATION TO STOCHASTIC PARTIAL DIFFERENTIAL EQUATIONS}

Using Theorems 2 we establish the stability for the family defined by the stochastic evolution equation of the form

$$
\mathrm{d} Z(t)=(A Z(t)+F(Z(t))) \mathrm{d} t+R \mathrm{~d} W(t) .
$$

Here we assume that $\mathcal{X}$ is a real separable Hilbert space, $A$ is the generator of a $C_{0^{-}}$ semigroup $S=(S(t))_{t \geq 0}$ acting on $\mathcal{X}, F$ is a mapping (not necessarily continuous) from $D(F) \subset \mathcal{X}$ to $\mathcal{X}, R$ is a bounded linear operator from another Hilbert space $\mathcal{H}$ to $\mathcal{X}$, and $W=(W(t))_{t \geq 0}$ is a cylindrical Wiener process on $\mathcal{H}$ defined over a certain filtered probability space $\left(\Omega, \mathcal{F},\left(\mathcal{F}_{t}\right)_{t \geq 0}, \mathbb{P}\right)$.

Let $Z_{0}$ be an $\mathcal{F}_{0}$-measurable random variable. By a solution of (3.1) starting from $Z_{0}$ we mean a solution to the stochastic integral equation (the so called mild solution)

$$
Z(t)=S(t) Z_{0}+\int_{0}^{t} S(t-s) F(Z(s)) \mathrm{d} s+\int_{0}^{t} S(t-s) R \mathrm{~d} W(s), \quad t \geq 0,
$$

see e.g. [3], where the stochastic integral appearing on the right hand side is understood in the sense of Itô. We suppose that for every $x \in \mathcal{X}$ there is a unique mild solution $Z^{x}=\left(Z_{t}^{x}\right)_{t \geq 0}$ of (3.1) starting from $x$, and that (3.1) defines in that way a Markov family. We assume that for any $x \in \mathcal{X}$, the process $Z^{x}(t), t \geq 0$ is stochastically continuous.

The corresponding transition semigroup is given by $P_{t} \psi(x)=\mathbb{E} \psi\left(Z^{x}(t)\right), t \geq 0, \psi \in$ $B_{b}(\mathcal{X}), x \in \mathcal{X}$. We assume that it is Feller.

A function $\Phi: \mathcal{X} \mapsto[0,+\infty)$ will be called a Lyapunov function, if it is measurable and

$$
\lim _{\|x\|_{\mathcal{X}} \rightarrow \infty} \Phi(x)=\infty .
$$


We shall assume that the deterministic equation

$$
\frac{\mathrm{d} Y(t)}{\mathrm{d} t}=A Y(t)+F(Y(t)), \quad Y(0)=x
$$

defines a continuous semi-dynamical system, i.e. for each $x \in \mathcal{X}$ there exists a unique continuous solution to (3.2) that we denote by $Y^{x}=\left(Y^{x}(t), t \geq 0\right)$ and for a given $t$ the mapping $x \mapsto Y^{x}(t)$ is measurable. Furthermore, we have $Y^{Y^{x}(t)}(s)=Y^{x}(t+s)$ for all $t, s \geq 0$ and $x \in \mathcal{X}$.

A set $\mathcal{K} \subset \mathcal{X}$ is called a global attractor for equation (3.2) if

- it is invariant under the semi-dynamical system generated by (3.2), i.e. $Y^{x}(t) \in \mathcal{K}$, $t \geq 0$ for any $x \in \mathcal{K}$,

- for any $\varepsilon, R>0$ there exists $T$ such that $Y^{x}(t) \in \mathcal{K}+\varepsilon B(0,1)$ for $t \geq T$ and $\|x\|_{\mathcal{X}} \leq R$.

If for any $\psi \in B_{b}(\mathcal{X})$ and $t \geq 0$ we have

$$
\int_{\mathcal{X}} \psi(x) \nu_{*}(\mathrm{~d} x)=\int_{\mathcal{X}} \psi\left(Y^{x}(t)\right) \nu_{*}(\mathrm{~d} x),
$$

we say that $\nu_{*} \in \mathcal{M}_{1}$ is invariant for (3.2).

The family $\left(Z^{x}(t)\right)_{t \geq 0}, x \in \mathcal{X}$, is stochastically stable if for every $\varepsilon, R, t>0$

$$
\inf _{x \in B(0, R)} \mathbb{P}\left(\left\|Z^{x}(t)-Y^{x}(t)\right\|_{\mathcal{X}}<\varepsilon\right)>0 .
$$

We derive from Theorem 2 the following result concerning stability of $Z$.

Theorem 3. Assume that:

- a global attractor $\mathcal{K}$ of the semi-dynamical system $\left(Y^{x}(t), t \geq 0\right)$ defined by (3.2) is a singleton,

- there exists a certain Lyapunov function $\Phi$ such that

$$
\sup _{t \geq 0} \mathbb{E} \Phi\left(Z^{x}(t)\right)<\infty, \quad \text { for any } x \in \mathcal{X},
$$

- the family $\left(Z^{x}(t)\right)_{t \geq 0}, x \in \mathcal{X}$, is stochastically stable and its transition semigroup has the e-property.

Then, the corresponding transition semigroup $\left(P_{t}\right)_{t \geq 0}$ is asymptotically stable.

Proof. Let $\mathcal{K}=\{z\}$. In [8] we have checked that $\left(P_{t}\right)_{t \geq 0}$ satisfies the assumption of Theorem 1 and $\mathcal{T}=\mathcal{X}$. To finish the proof it is enough to show that condition (2.24) holds. Fix $\delta>0$. Since $\sup _{t>0} \mathbb{E}\left(\Phi\left(Z^{z}(t)\right)\right)<+\infty$ for some Lyapunov function $\Phi$, there exists a bounded Borel set $\bar{D} \subset \mathcal{X}$ such that

$$
P_{t}^{*} \delta_{z}(D)>\frac{1}{2} \quad \text { for } t \geq 0
$$


On the other hand, from the fact that the family $\left(Z^{x}(t)\right)_{t \geq 0}, x \in \mathcal{X}$, is stochastically stable we have

$$
\inf _{x \in D} \mathbb{P}\left(\left\|Z^{x}(t)-Y^{x}(t)\right\|_{\mathcal{X}}<\varepsilon\right)>0 \quad \text { for any } t, \varepsilon>0 .
$$

Let $t_{0}>0$ be such that $Y^{x}\left(t_{0}\right) \in B(z, \delta / 2)$ for $x \in D$ and let $\varepsilon=\delta / 2$. Then

$$
\gamma:=\inf _{x \in D} \mathbb{P}\left(\left\|Z^{x}\left(t_{0}\right)-z\right\|_{\mathcal{X}}<\delta\right)>0
$$

Consequently,

$$
\begin{aligned}
& \liminf _{t \rightarrow \infty} P_{t}^{*} \delta_{z}(B(z, \delta))=\liminf _{t \rightarrow \infty} P_{t+t_{0}}^{*} \delta_{z}(B(z, \delta))=\liminf _{t \rightarrow \infty} \int_{\mathcal{X}} \mathbf{1}_{B(z, \delta)}(x) P_{t+t_{0}}^{*} \delta_{z}(\mathrm{~d} x) \\
& =\liminf _{t \rightarrow \infty} \int_{\mathcal{X}} P_{t_{0}} 1_{B(z, \delta)}(x) P_{t}^{*} \delta_{z}(\mathrm{~d} x)=\liminf _{t \rightarrow \infty} \int_{\mathcal{X}} P_{t_{0}}^{*} \delta_{x}(B(z, \delta))(x) P_{t}^{*} \delta_{z}(\mathrm{~d} x) \\
& \geq \liminf _{t \rightarrow \infty} \int_{D} P_{t_{0}}^{*} \delta_{x}(B(z, \delta))(x) P_{t}^{*} \delta_{z}(\mathrm{~d} x) \geq \gamma \liminf _{t \rightarrow \infty} P_{t}^{*} \delta_{z}(D)>\gamma / 2 .
\end{aligned}
$$

This completes the proof.

\section{Application to the Passive Tracer Model}

In the last section we are concerned with some model of transport of a passive tracer in a compressible random flow (see [5, 6, 7]). Applying Theorem 3 we will be able to prove that the distribution of velocity of a particle is weakly convergent to some distribution. This result is in the same spirit as results in [8], where was derived the weak law of large numbers for the trajectory of a particle described by the model mentioned above.

Let us consider the ordinary differential equation

$$
\frac{\mathrm{d} \mathbf{x}(t)}{\mathrm{d} t}=V(t, \mathbf{x}(t)), \quad \mathbf{x}(0)=\mathbf{x}_{0}
$$

where $V$ is the random field. We assume that this field is spatially periodic, i.e.

$$
V\left(t, x+2 \pi \epsilon_{j}\right)=V(t, x), \quad \forall t \geq 0, x \in \mathbb{R}^{d}, j=1, \ldots, d,
$$

where $\epsilon_{j}=\underbrace{(0, \ldots, 1, \ldots, 0)}_{j \text {-th positon }}, j=1, \ldots, d$ are unit versors in $\mathbb{R}^{d}$. It is additionally zero mean Gaussian, with the covariance matrix

$$
R_{i, j}(t-s, x-y):=\mathbb{E}\left[V_{i}(t, x) V_{j}(s, y)\right]
$$

whose Fourier transform in the $x$-variable is given by

$$
\hat{R}_{i, j}(h, k):=\frac{1}{(2 \pi)^{d}} \int_{\mathbb{T}^{d}} \mathrm{e}^{-\mathrm{i} k x} R_{i, j}(h, x) \mathrm{d} x=\mathrm{e}^{-\gamma(k)|h|} \mathcal{E}_{i, j}(k), i, j=1, \ldots, d, k \in \mathbb{Z}^{d} .
$$

Here $\mathbb{T}^{d}:=[0,2 \pi)^{d}$. Let $S_{+}(d)$ denote the space of all non-negative definite, Hermitian matrices. We assume that the energy spectrum $\mathcal{E}:=\left[\mathcal{E}_{i, j}\right]: \mathbb{Z}^{d} \mapsto S_{+}(d)$ and mixing rates 
$\gamma: \mathbb{Z}^{d} \mapsto(0,+\infty)$ satisfy the following assumption: there exist $m>d / 2+1$ and $\alpha \in(0,1)$ such that

$$
\||\mathcal{E}|\|^{2}:=\sum_{k \in \mathbb{Z}^{d}}|\gamma(k)|^{\alpha}|k|^{2(m+1)} \operatorname{Tr} \mathcal{E}(k)<+\infty
$$

Moreover,

$$
\begin{gathered}
\inf _{k \in \mathbb{Z}_{*}^{d}} \gamma(k)=\gamma_{*}>0, \\
\lim _{|k| \rightarrow+\infty} \gamma(k)=0
\end{gathered}
$$

and

$$
\int_{0}^{\infty} \sup _{k \in \mathbb{Z}_{*}^{d}} \mathrm{e}^{-\gamma(k) t}|k| \mathrm{d} t<\infty .
$$

Here $\operatorname{Tr} \mathcal{A}$ denotes a trace of a given matrix $\mathcal{A}$. We will also need the following nondegeneracy assumptions of the spectrum

$$
\forall k \in \mathbb{Z}_{*}^{d}, \quad \operatorname{det} \mathcal{E}(k) \neq 0 .
$$

Given $r \geq 0$ we denote by $\mathcal{H}^{r}$ the Sobolev space which is the completion of

$$
\left\{\xi \in C^{\infty}\left(\mathbb{T}^{d} ; \mathbb{R}^{d}\right): \int_{\mathbb{T}^{d}} \xi(x) \mathrm{d} x=0\right\}
$$

with respect to the norm $\|\xi\|_{\mathcal{H}^{r}}^{2}:=\sum_{k \in \mathbb{Z}_{*}^{d}}|k|^{2 r}|\widehat{\xi}(k)|^{2}$, where $\hat{\xi}(k):=(2 \pi)^{-d} \int_{\mathbb{T}^{d}} \xi(x) e^{-i x \cdot k} \mathrm{~d} x$, $k \in \mathbb{Z}^{d}$ are the Fourier coefficients of $\xi$. Note that obviously $\mathcal{H}^{u} \subset \mathcal{H}^{r}$ if $u>r$.

Let $A_{r}$ be an operator on $\mathcal{H}^{r}$ defined by

$$
\widehat{A_{r} \xi}(k):=-\gamma(k) \widehat{\xi}(k), \quad k \in \mathbb{Z}_{*}^{d},
$$

with the domain

$$
D\left(A_{r}\right):=\left\{\xi \in \mathcal{H}^{r}: \sum_{k \in \mathbb{Z}_{*}^{d}}|\gamma(k)|^{2}|k|^{2 r}|\widehat{\xi}(k)|^{2}<\infty\right\} .
$$

Since the operator is self-adjoint it generates a $C_{0}$-semigroup $\left(S_{r}(t)\right)_{t \geq 0}$ on $\mathcal{H}^{r}$. Moreover, for $u>r, A_{u}$ is the restriction of $A_{r}$ and $S_{u}$ is the restriction of $S_{r}$. From now on, we will omit the subscript $r$ writing $A$ and $S$ instead of $A_{r}$ and $S_{r}$.

Let $Q$ be a symmetric positive-definite bounded linear operator on $L_{0}^{2}:=\mathcal{H}^{0}$ given by

$$
\widehat{Q \xi}(k):=\gamma(k) \mathcal{E}(k) \widehat{\xi}(k), \quad k \in \mathbb{Z}_{*}^{d} .
$$

Let $m$ be the constant appearing in (4.2) and let $\mathcal{H}:=\mathcal{H}^{m}$ and $\mathcal{V}:=\mathcal{H}^{m+1}$. Note that by Sobolev embedding, see e.g. Theorem 7.10, p. 155 of [4], there exists a constant $C>0$ such that

$$
\|\xi\|_{C^{1}\left(\mathbb{T}^{d} ; \mathbb{R}^{d}\right)} \leq C\|\xi\|_{\mathcal{H}}, \quad \forall \xi \in \mathcal{H}
$$


Let $Z(s, x):=V(s, x+\mathbf{x}(s))$ be the Lagrangian observation of the environment process, or shortly the observation process. It is known, see [6] and [7], that $Z(s, \cdot)$ solves the equations

$$
\mathrm{d} Z(t)=[A Z(t)+B(Z(t), Z(t))] \mathrm{d} t+Q^{1 / 2} \mathrm{~d} W(t), \quad Z(0, \cdot)=V(0, \mathbf{x}(0)+\cdot) .
$$

where $W$ is a certain cylindrical Wiener process on some probability space $\mathfrak{A}(\mathfrak{A}=$ $\left.\left(\Omega, \mathcal{F},\left(\mathcal{F}_{t}\right)_{t \geq 0}, \mathbb{P}\right)\right)$ and

$$
B(\psi, \xi)(x):=\left(\sum_{j=1}^{d} \psi_{j}(0) \frac{\partial \xi_{1}}{\partial x_{j}}(x), \ldots, \sum_{j=1}^{d} \psi_{j}(0) \frac{\partial \xi_{d}}{\partial x_{j}}(x)\right), \quad \psi, \xi \in \mathcal{H}, x \in \mathbb{T}^{d} .
$$

By $(4.10), B(\cdot, \cdot)$ is a continuous bilinear form acting from $\mathcal{H} \times \mathcal{H}$ into $\mathcal{H}^{m-1}$.

Given $\xi \in \mathcal{H}$ let $\left(Z^{\xi}(t)\right)_{t \geq 0}$ denote the value at $t \geq 0$ of a solution to (4.11) satisfying $Z^{\xi}(0, x)=\xi(x)$ for $x \in \mathbb{T}^{d}$. As usually, the transition semigroup is given by the formula: $P^{t} \psi(\xi):=\mathbb{E} \psi\left(V_{\xi}\left(t, \mathbf{x}_{\xi}(t)+\cdot\right)\right)$ for $\psi \in \mathcal{H}$. It is known (see for instance [3]) that the Feller property and stochastic continuity hold for the transition semigroup. Moreover, it has been proved, see [8], that this semigroup satisfies the assumptions of Theorem 3. Having this we were able to derive that the Markov family $\left(Z^{\xi}(t)\right)_{t \geq 0}$ is mean * ergodic (see $[8,14]$ ). Applying Theorem 3 we obtain, in turn, that the transition semigroup $\left(P_{t}\right)_{t \geq 0}$ is asymptotically stable.

We are in a position to formulate the following proposition.

Proposition 4. Under assumptions (4.2) - (4.6) there exists a distribution $\mathbb{V}$ such that

$$
\underset{s \rightarrow \infty}{\mathrm{W}-\lim _{s \rightarrow \infty}} \frac{\mathrm{d} x}{\mathrm{~d} t}(s)=\mathbb{V} \text {. }
$$

Proof. From the fact that $\left(P_{t}\right)_{t \geq 0}$ is asymptotically stable it follows that the distribution $\mathcal{L}(Z(s, x))$ of the vector $Z(s, x)$ tends weakly to $\mu_{*}$, where $\mu_{*}$ denotes the unique invariant measure of $\left(P_{t}\right)_{t \geq 0}$. Let $r: \mathcal{H} \rightarrow \mathbb{R}$ denote the function given by the formula $r(x)=x(0)$. From condition (4.10) we immediately obtain that $r$ is continuous. Hence $\mathcal{L}(r(Z(s, x)))$ converges weakly to $\mu_{*} \circ r^{-1}$. Let $\mathbb{V}=\mu_{*} \circ r^{-1}$. Observation that $r(Z(s, x))=Z(s, 0)=$ $V(s, x(s))=\frac{\mathrm{d} x}{\mathrm{~d} t}(s)$ finishes the proof.

\section{REFERENCES}

[1] Billingsley, P., Convergence of probability measures, 2-nd ed. Wiley \& Sons, New York, 1999.

[2] Dudley, R. M. Probabilities and Matrics, Aarhus Universitet, 1976.

[3] Da Prato, G. and Zabczyk, J., Stochastic Equations in Infinite Dimensions, Cambridge University Press, Cambridge 1992.

[4] Gilbarg, D. and Trudinger N. S., Elliptic Partial Differential Equations of Second Order, SpringerVerlag, Berlin, Heidelberg, New York, 1983.

[5] Fannjiang, A. and Komorowski, T., Turbulent Diffusion in Markovian Flows, Ann. of Appl. Prob. 9, 591-610, (1999).

[6] Fannjiang, A., Komorowski, T., and Peszat, S., Lagrangian dynamics for a passive tracer in a class of Gaussian Markovian flows, Stoch. Proc. Appl. 97, (2002) 171-198.

[7] Komorowski, T. and Peszat, S., Transport of a passive tracer by an irregular velocity field, J. Statist. Phys. 115 (2004), 1383-1410. 
[8] Komorowski, T., Peszat, S., and Szarek, T., Ergodicity of some Markov processes, Ann. of Prob. (to appear), http://arxiv.org/abs/0810.4609, 2008.

[9] Lasota, A. and Mackey, M. C., Chaos, Fractals, and Noise, Springer-Verlag, 1994.

[10] Lasota, A. and Yorke J. A., On the existence of invariant measures for piecewise monotonic transformations, Trans. Amer. Math. Soc. 186 (1973), 481-488.

[11] Lasota, A. and Yorke J. A., Lower bound technique for Markov operators and iterated function systems, Random \& Computational Dynamics 2 (1994), 41-77.

[12] Lasota, A. and Szarek, T., Lower bound technique in the theory of a stochastric differential equation, J. Differential Equations 231, (2006), 513-533.

[13] Szarek, T., The uniqueness of invariant measures for Markov operators, Studia Math. 189 (3) (2008), $225-233$

[14] Zaharopol, R., Invariant Probalbilities of Markov-Feller Operators and Their Supports, Birkhäuser Verlag AG, (2005).

Institute of Mathematics, University of Silesia, Bankowa 14, 40-007 Katowice, Poland E-mail address: szarek@intertele.pl

Institute of Mathematics, University of Silesia, Bankowa 14, 40-007 Katowice, Poland E-mail address: sleczka@ux2.math.us.edu.pl

Department of Mathematics, University of North Texas, P.O. Box 311430 Denton, tX 76203-1430, USA

E-mail address: urbanski@unt.edu 